\title{
Glutathione Precursor, N-Acetyl-Cysteine, Improves Mismatch Negativity in Schizophrenia Patients
}

\author{
Suzie Lavoie ${ }^{1,2,11}$, Micah M Murray3,4,5, I', Patricia Deppen ${ }^{1,2}$, Maria G Knyazeva ${ }^{4}$, Michael Berk ${ }^{6,7}$, \\ Olivier Boulat ${ }^{8}$, Pierre Bovet ${ }^{2}$, Ashley I Bush ${ }^{6}$, Philippe Conus ${ }^{2}$, David Copolov', Eleonora Fornari ${ }^{4}$, \\ Reto Meuli ${ }^{4}$, Alessandra Solida ${ }^{2}$, Pascal Vianin'2, Michel Cuénod ${ }^{1,2}$, Thierry Buclin ${ }^{10}$ and Kim $\mathbf{Q} \mathbf{D o}^{*, 1,2}$ \\ 'Center for Psychiatric Neuroscience, Centre Hospitalier Universitaire Vaudois and University of Lausanne, Switzerland; ${ }^{2}$ Department of \\ Psychiatry, Centre Hospitalier Universitaire Vaudois and University of Lausanne, Lausanne, Switzerland; ${ }^{3} E E G$ Core, Center for Biomedical \\ Imaging of Lausanne and Geneva, Centre Hospitalier Universitaire Vaudois and University of Lausanne, Switzerland; ${ }^{4}$ Radiology Service, Centre \\ Hospitalier Universitaire Vaudois and University of Lausanne, Lausanne, Switzerland; ${ }^{5}$ Functional Electrical Neuroimaging Laboratory, \\ Neuropsychology and Neurorehabilitation Service, Centre Hospitalier Universitaire Vaudois and University of Lausanne, Lausanne, Switzerland; \\ ${ }^{6}$ Mental Health Research Institute of Victoria, Victoria, Australia; ${ }^{7}$ Department of Clinical and Biomedical Sciences, University of Melbourne, \\ Melbourne, Australia; ${ }^{8}$ Central Laboratory for Clinical Chemistry, Centre Hospitalier Universitaire Vaudois and University of Lausanne, Lausanne, \\ Switzerland; ${ }^{9}$ Monash University, Clayton, Australia; ${ }^{10}$ Department of Clinical Pharmacology, Centre Hospitalier Universitaire Vaudois and \\ University of Lausanne, Lausanne, Switzerland
}

\begin{abstract}
In schizophrenia patients, glutathione dysregulation at the gene, protein and functional levels, leads to N-methyl-D-aspartate (NMDA) receptor hypofunction. These patients also exhibit deficits in auditory sensory processing that manifests as impaired mismatch negativity $(\mathrm{MMN})$, which is an auditory evoked potential (AEP) component related to NMDA receptor function. N-acetyl-cysteine (NAC), a glutathione precursor, was administered to patients to determine whether increased levels of brain glutathione would improve MMN and by extension NMDA function. A randomized, double-blind, cross-over protocol was conducted, entailing the administration of NAC (2g/day) for 60 days and then placebo for another 60 days (or vice versa). 128-channel AEPs were recorded during a frequency oddball discrimination task at protocol onset, at the point of cross-over, and at the end of the study. At the onset of the protocol, the MMN of patients was significantly impaired compared to sex- and age- matched healthy controls $(p=0.003)$, without any evidence of concomitant P300 component deficits. Treatment with NAC significantly improved MMN generation compared with placebo $(p=0.025)$ without any measurable effects on the P300 component. MMN improvement was observed in the absence of robust changes in assessments of clinical severity, though the latter was observed in a larger and more prolonged clinical study. This pattern suggests that MMN enhancement may precede changes to indices of clinical severity, highlighting the possible utility AEPs as a biomarker of treatment efficacy. The improvement of this functional marker may indicate an important pathway towards new therapeutic strategies that target glutathione dysregulation in schizophrenia.

Neuropsychopharmacology (2008) 33, 2187-2199; doi: I0.1038/sj.npp. I301624; published online I4 November 2007
\end{abstract}

Keywords: schizophrenia; glutathione; auditory evoked potential; mismatch negativity; NMDA receptors; N-acetyl-cysteine

\section{INTRODUCTION}

The pathophysiology of schizophrenia is thought to entail deficits at anatomical, genetic, and functional levels. Of growing interest is the observation of decreased glutathione (GSH) levels in cerebrospinal fluid, prefrontal cortex (Do et al, 2000), and post-mortem caudate (Yao et al, 2006) of

*Correspondence: Dr KQ Do, Center for Psychiatric Neuroscience, Department of Psychiatry, Lausanne University Hospital, Prilly 1008, Switzerland,

Tel: +4I 21643 6565, Fax: +4I 216436562 ,

E-mail: Kim.Do@CHUV.ch

"These two authors have contributed equally to this work.

Received 10 May 2007; accepted 9 October 2007 schizophrenia patients. Evidence indicates that there is a defect in GSH synthesis at the level of the key synthesizing enzyme, glutamate cysteine ligase. Polymorphisms in the gene of the modifier subunit of this enzyme as well as decreased expression of this gene have been associated with schizophrenia (Do et al, in press; Tosic et al, 2006; Gysin et al, 2007). GSH is a major antioxidant and redox regulator that protects cells against oxidative stress (Meister and Anderson, 1983). Through its function as a reducing agent, GSH can potentiate the activity of redox sensitive proteins, such as $N$-methyl-D-aspartate (NMDA) receptors (Choi and Lipton, 2000; Kohr et al, 1994). During oxidative stress, GSH is converted to its oxidized form and can additionally impact NMDA receptor activity when it is transported 
extracellularly (Janaky et al, 1993; Sucher and Lipton, 1991). Animal models in which GSH levels are decreased show hypofunction of NMDA receptors (Steullet et al, 2006). During development, the transitory decrease of GSH levels in conjunction with an increase in dopamine result in morphological (Cabungcal et al, 2006; Gheorghita et al, 2007, unpublished work) and behavioral (Cabungcal et al, 2007; Castagné et al, 2004a, b) impairments in animals that are similar to those observed in schizophrenia (Garey et al, 1998; Glantz and Lewis, 2000; Kolluri et al, 2005; Robbins, 2005). Several studies have demonstrated that the administration of NMDA receptor antagonists, such as phencyclidine or ketamine, induces in control subjects symptoms that are similar to those observed in schizophrenia and exacerbates these symptoms in patients (Krystal et al, 1994). It has been proposed that impairments in NMDA receptor activity may contribute to the pathophysiology of schizophrenia (Coyle, 2006; Javitt and Zukin, 1991). Collectively, these lines of evidence support the hypothesis that the dysregulation of the GSH system reduces activity of NMDA receptors in schizophrenia.

Deficits in NMDA receptor function can be quantitatively and non-invasively assessed through the measurement of auditory evoked potentials (AEPs) and in particular the mismatch negativity (MMN). The MMN is an AEP component pre-attentively elicited by discriminable stimulus changes in an otherwise contiguous stream of events that typically peaks $\sim 150-200 \mathrm{~ms}$ post-stimulus onset (Näätänen et al, 1978). NMDA receptor antagonists block MMN generation in both primates (Javitt et al, 1996) and humans (Umbricht et al, 2000), suggesting that the MMN reflects current flow through NMDA channels. The amplitude of MMN is known to be decreased in schizophrenia (Catts et al, 1995; Javitt et al, 1993, 1998; Shelley et al, 1991; Shutara et al, 1996; for review see Turetsky et al, 2007), further implicating impaired NMDA function in such patients.

In the present study, we investigated whether increasing brain levels of GSH in schizophrenia patients would improve cerebral functioning and in particular MMN generation (Light and Braff, 2005b). Cysteine is the limiting precursor in the synthesis of GSH (Meister et al, 1986), but is not an ideal delivery system to the cell as it is potentially toxic and is spontaneously catabolized in the gastrointestinal tract and blood plasma. $\mathrm{N}$-acetyl-cysteine (NAC) has therefore been used in various studies as a cysteine donor. Given orally, NAC is quickly absorbed, and peak plasma concentration of cysteine is reached within $120 \mathrm{~min}$ (Borgstrom et al, 1986; Borgstrom and Kagedal, 1990; Olsson et al, 1988). NAC crosses the blood-brain barrier (Farr et al, 2003), and cysteine can be used in the brain as a GSH precursor. Animal studies have indeed shown that systemic administration of NAC protects the brain against GSH depletion (Aydin et al, 2002; Ercal et al, 1996; Fu et al, 2006; Kamboj et al, 2006).

NAC was used to increase GSH levels in the context of a larger multi-center clinical trial. Effects of GSH modulation on the psychopathological status of patients were separately assessed by Berk et al (2007, unpublished work). The present study instead focuses on the objective effects of NAC on the MMN, as well as on the GSH-related thiols metabolic status of our subset of schizophrenia patients. We show that NAC improves MMN in schizophrenia patients.

\section{MATERIALS AND METHODS}

\section{Clinical Trial Protocol}

NAC (1 g $2 \times /$ day $)$ and placebo were administered to schizophrenia patients in a double-blinded, crossover design. The clinical trial was conducted from November 2003 to November 2005. One group received NAC for the first 2 months and then placebo for another period of 2 months, and the other group received placebo first and then NAC. NAC was purchased from Zambon (Italy). NAC and placebo capsules were manufactured by DFC Thompson (Sydney, Australia) and re-conditioned by a pharmacist of the Department of Psychiatry of the Centre Hospitalier Universitaire Vaudois and University of Lausanne. NAC being a precursor of GSH, we hypothesized that increasing brain levels of GSH in schizophrenia patients would improve cerebral functioning and in particular MMN generation. Electroencephalographic (EEG) recordings and blood sampling were performed at the onset of the protocol (baseline measurements), at the point of crossover, and at the end of the study. Psychopathological scales were evaluated every 2 weeks, according to the main trial protocol; the results of which are reported elsewhere (Berk et al, 2007, unpublished work).

\section{Participants}

Eleven patients (nine men; two women; aged $31.9 \pm 3.4$ years; mean \pm SEM) meeting DSM-IV criteria for schizophrenia were recruited from the ambulatory Schizophrenia Service of the Department of Psychiatry of the Centre Hospitalier Universitaire Vaudois by an experienced psychiatrist and a psychologist well trained in Diagnostic Interview for Genetic Studies. The mean duration of illness was $9.4 \pm 2.5$ years. Data from these patients at the onset of the protocol were compared with those from 11 sexmatched and age-matched healthy controls (nine men; two women; aged $34.4 \pm 2.9$ years), who were selected on the basis that they had never suffered from any psychiatric disorder.

Among the 11 patients, 9 participated in the clinical trial and 7 completed the entire study, including EEG recordings at crossover and at the end of the study (see Table 1). The two patients who withdrew from the study reported that it was too demanding for them. Of the seven patients who completed the entire study, five were among the group that first received NAC and then placebo; the remaining two received placebo first and then NAC. As such, any effects observed during NAC administration are unlikely to follow simply from repeated task performance. Following their recruitment, patients were given an ID number, and both patients and investigators were blinded until the time of analysis, when data pooling necessitated unblinding. Patients and controls were recruited with fully informed written consent, and all procedures were approved by the Ethics Committee of the Faculty of Biology and Medicine of the University of Lausanne. All 11 patients and 8 of the healthy controls were interviewed with the Diagnostic Interview for Genetic Studies, developed by the NIMH (Nurnberger et al, 1994; Preisig et al, 1999). 
Table I Demographic and Clinical Characteristics of Participants

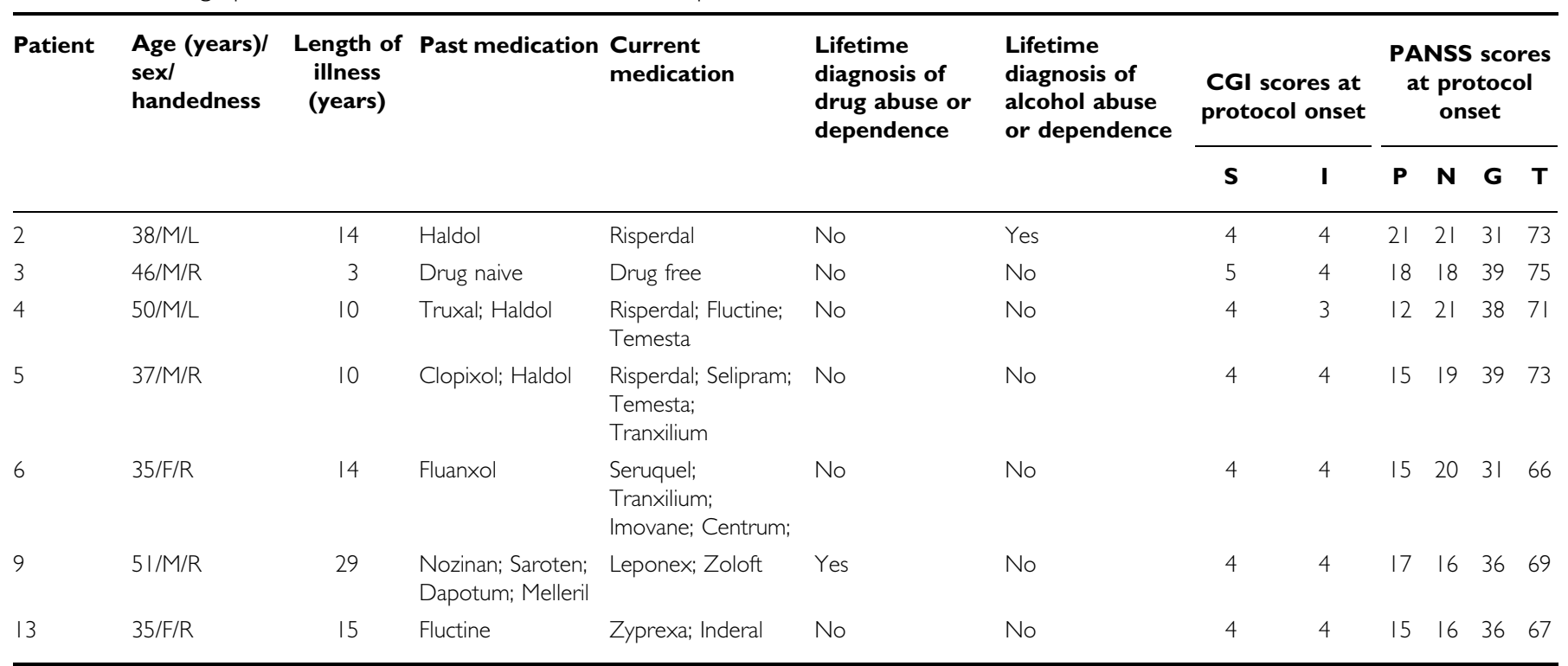

CGI and PANSS scales: S, severity; I, improvement; P, positive; N, negative; G, general psychopathology; T, total score.

\section{EEG Experimental Paradigm}

During the EEG recordings, participants were presented with auditory pure tone stimuli $(50 \mathrm{~ms}$ duration; $10 \mathrm{~ms}$ rise/ fall time; $44.1 \mathrm{kHz}$ sampling rate; $70 \mathrm{~dB}$ SPL at the ear) and performed a go/no-go oddball discrimination task. On the majority of trials $(80 \%)$, the pitch was $1 \mathrm{kHz}$, and on the remaining $20 \%$ it was $2 \mathrm{kHz}$. Stimuli were presented binaurally via insert earphones (model ER-4P; Etymotic Research Inc., Elk Grove Village, IL), and the inter-stimulus interval was $1.5 \mathrm{~s}$. Participants were instructed to perform a button press upon hearing the $2 \mathrm{kHz}$ tone (deviant) and to withhold responses to the $1 \mathrm{kHz}$ tone (standard). Stimulus presentation and response recording were controlled by E-prime (Psychology Software Tools Inc., Pittsburgh).

\section{EEG Recordings and Analyses}

Continuous EEG was recorded at $500 \mathrm{~Hz}$ (baseline measurements) or $1000 \mathrm{~Hz}$ (crossover and at the end of the protocol) through a 128-channel Geodesic Sensor Net system (Electrical Geodesics Inc., Eugene, OR) referenced to the vertex $(\mathrm{Cz})$ and $100 \mathrm{~Hz}$ low-pass filtered. Electrode impedance was maintained below $50 \mathrm{k} \Omega$. Peri-stimulus epochs of continuous EEG ( $100 \mathrm{~ms}$ before to $600 \mathrm{~ms}$ after stimulus onset) were averaged from each participant separately for the two different stimuli (ie standards and deviants) to compute AEPs. Epochs with muscle activity, eye movements, or other noise transients exceeding $\pm 100 \mu \mathrm{V}$ were automatically rejected off-line. Data from artifact electrodes were interpolated (Perrin et al, 1987), and AEPs were downsampled to a common 121-channel montage and baselinecorrected using the $100 \mathrm{~ms}$ pre-stimulus period. AEPs were then recalculated to the common average reference and group-averaged.

For baseline recordings, AEPs from control subjects were based on $316 \pm 24$ (mean \pm SEM) standard and $73 \pm 5$ deviant stimuli, whereas those from patients were based on $590 \pm 57$ standard and $148 \pm 14$ deviant stimuli. Patients were presented with a larger number of stimuli to minimize the possibility that differences between populations followed from a lower signal-to-noise ratio in patients. The number of trials accepted in response to standard and deviant stimuli did not differ between recordings during NAC and placebo treatments. Following NAC treatment, $726 \pm 39$ standard and $179 \pm 9$ deviant trials were included. Following placebo treatment, $626 \pm 95$ standard and $164 \pm 18$ deviant trials were included.

Two separate series of AEP analyses were conducted. The first followed a between-subject designs and was carried out to verify that our cohort of patients exhibited impaired MMN relative to healthy controls at the onset of the protocol, as has been repeatedly demonstrated in prior studies of schizophrenic patients (Catts et al, 1995; Javitt et al, 1993, 1998; Shelley et al, 1991; Shutara et al, 1996). The second followed a within-subject designs and was carried out to assess whether 2 months of NAC administration restored MMN generation relative to the administration of placebo. AEPs were analyzed following methods described in detail elsewhere that permit the examination of both local and global features of the electric field at the scalp (Murray et al, 2004). We briefly summarize these methods here.

First, AEP components were identified using a topographic pattern analysis that is based on a modified atomize and agglomerate hierarchical clustering procedure as implemented in Cartool software (http://brainmapping. unige.ch/cartool.htm; see also Tibshirani et al, 2005). This clustering procedure identifies the topographies (ie maps) dominating the group-averaged AEPs across populations/ conditions. The pattern observed at the group-average level was then statistically assessed at the individual participant level using a fitting procedure based on spatial correlation (Brandeis et al, 1995; see also Murray et al, 2006, for a recent publication of formulae), yielding a measure of map presence. These values are then submitted to ANOVA, revealing whether and when different maps explain 
responses from different populations/conditions and by extension whether and when different configurations of underlying sources are active, because topographic changes forcibly follow from different configurations of underlying brain networks (Lehmann, 1987). As this analysis identified the same set and sequence of topographies in AEPs from both patient and control groups as well as all experimental conditions, we do not discuss it in detail in the Results section. The main utility of the topographic pattern analysis in the present study was in identifying the onset and offset of AEP components according to topographic stability in time (see Results section for details). In particular, we focus on the $\mathrm{N} 1$ component, which in all cases was identified over the $70-155 \mathrm{~ms}$ interval, given the evidence that the MMN is contemporaneous in time with the $\mathrm{N} 1$ when standard and deviant stimuli are highly divergent (although the MMN generators are thought to be distinct). In addition to AEP waveforms at specific electrode locations (which were selected based on the AEP topography and using sites common to prior studies), we also analyzed the strength of the electric field at the scalp using global field power (GFP; Lehmann and Skrandies, 1980). GFP is equivalent to the root mean square across the electrode montage. AEP and GFP waveform area measures ( $v s$ the $0 \mu \mathrm{V}$ baseline) from each stimulus condition over the $70-155 \mathrm{~ms}$ interval were compared between controls and patients at protocol onset, and between NAC and placebo treatments.

We estimated the sources in the brain underlying the AEPs from each treatment type and stimulus condition using the LAURA distributed linear inverse solution (Grave de Peralta Menendez et al, 2001, 2004; see Michel et al, 2004 for a comparison of inverse solution methods as well as a discussion of the benefits of high-density electrode montages for this purpose). LAURA selects the source configuration that better mimics the biophysical behavior of electric vector fields (ie activity at one point depends on the activity at neighboring points according to electromagnetic laws based on the Maxwell equations). The solution space was calculated on a realistic head model that included 4024 nodes, selected from a $6 \times 6 \times 6 \mathrm{~mm}$ grid equally distributed within the gray matter of the Montreal Neurological Institute's average brain. The results of the above topographic pattern analysis defined time periods for which intracranial sources were estimated. We would remind the reader that the source estimations presented here provide a visualization of the likely generators and do not represent a statistical analysis.

\section{Biochemical Measurements and Analyses}

GSH levels were measured in blood cells and GSH-related thiols, cysteine and cysteinyl-glycine, were measured in plasma. Blood was collected by venipuncture between 0700 and 0830 hours under restricted activity conditions and after fasting from the previous midnight. About $18-20 \mathrm{ml}$ blood was collected in Vacutainer tubes coated with ethylenediaminetetraacetic acid (Becton Dickinson, Franklin Lake, NJ). Hemoglobin was quantified before blood centrifugation for $5 \mathrm{~min}$ at $3000 \mathrm{~g}$ and $4^{\circ} \mathrm{C}$. The pellet, corresponding to blood cells, was washed twice with $0.9 \%$ $\mathrm{NaCl}$ and frozen at $-80^{\circ} \mathrm{C}$ until analyses. The supernatant, corresponding to the plasma, was sampled in aliquots and kept at $-80^{\circ} \mathrm{C}$ until analyses. Total GSH in blood cells was determined using a diagnostic kit purchased by Calbiochem (EMD Biosciences Inc., Darmstadt, Germany) and is expressed in $\mu \mathrm{mol} \mathrm{GSH}$ per $\mathrm{ml}$ of blood. The method is based on a colorimetric assay of a chromophoric thione formed specifically between the reagent and GSH. Total cysteine and cysteinyl-glycine were quantified in plasma with a technique adapted from (Jacobsen et al, 1994). Briefly, thiols were reduced and/or decoupled from proteins by reaction with Tris (2-carboxyethyl) phosphine (Krijt et al, 2001). After deproteinization with perchloric acid, thiols were derivatized with 7-fluorobenzofurazane-4-sulfonic acid (SBD-F). SBD-F derivatives were analyzed by HPLC followed by fluorometric detection. Concentrations are expressed in $\mu \mathrm{mol} / \mathrm{l}$. From blood samples, analyses were primarily focused on GSH levels in blood as well as on levels of precursors: cysteine, one of the substrates of GCL, and cysteinyl-glycine, the product of GCL. These measures were assessed by a two-way mixed effects ANOVA for crossover design with terms for Period (sequence), Patient and Treatment. The relevant effect of the treatment was adjusted for the random effect of the patient and for the study period (linked to the treatment sequence).

\section{RESULTS}

\section{Baseline Measurements: Controls vs Patients}

Behavioral results. Reaction times on the auditory discrimination task did not differ between patients (mean \pm $\mathrm{SEM}=772 \pm 45 \mathrm{~ms})$ and healthy controls $(610 \pm 77 \mathrm{~ms})$. Likewise, performance accuracy was near ceiling levels for both groups (patients: $98 \pm 1 \%$; controls: $100 \pm 0 \%$ ). This pattern of results thus argues against differences in selective attention or task performance as underlying AEP differences between groups.

Electrophysiological results. The topographic pattern analysis identified the same sequence of maps in both populations and thus provided no evidence for differences in the configuration of underlying brain networks either between conditions or populations. The results of that analysis will therefore not be discussed further. However, this method of defining AEP components provided a more objective means for defining time periods for the analysis of AEP and GFP waveforms. Likewise, the topography of the AEP (ie the location of peak amplitude) was used for the selection of specific scalp electrodes for the analysis of AEP waveforms. The N1 component of the AEP was identified over the $70-155 \mathrm{~ms}$ interval.

AEP waveforms from four fronto-central electrodes are displayed in Figure 1a. Visual inspection of these waveforms indicates that healthy controls showed an early differential response (ie the MMN) between standard and deviant stimuli, whereas patients did not. This pattern is in keeping with what would be expected from prior investigations of the MMN in schizophrenia patients (Catts et al, 1995; Javitt et al, 1993, 1998; Shelley et al, 1991; Shutara et al, 1996). To statistically assess whether our cohort of patients exhibited a deficient MMN, area measures ( $v s$ the $0 \mu \mathrm{V}$ baseline) were taken from these electrodes over the 70-155 ms interval and submitted to a $2 \times 2 \times 4$ repeated measures ANOVA, using 
a Exemplar AEP waveforms (Baseline)
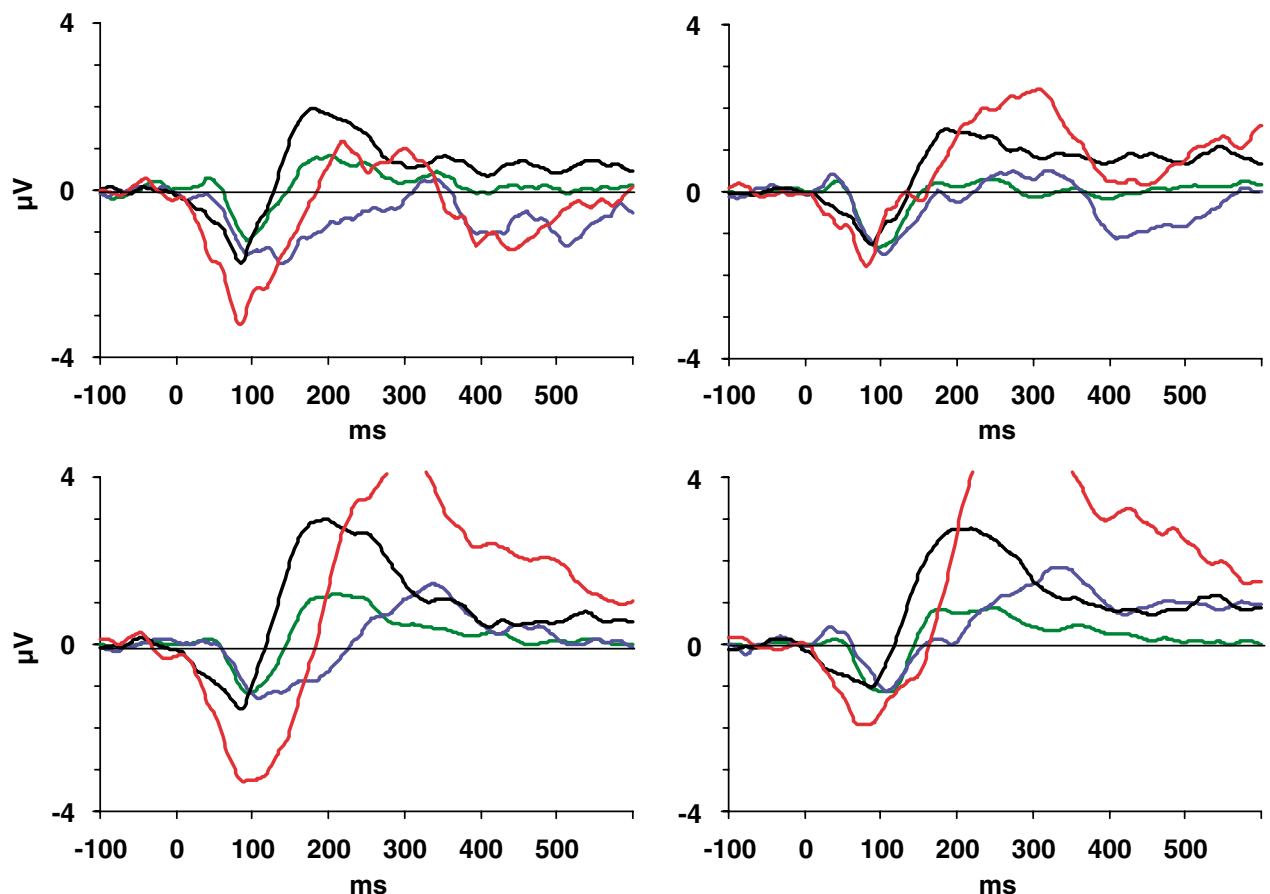

Mean MMN area (70-155ms) across electrodes

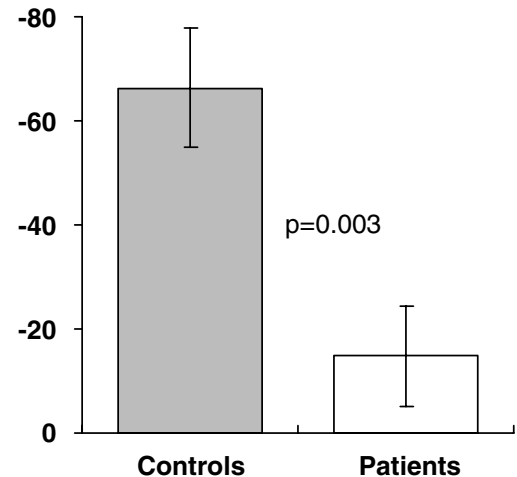

Controls (deviant)

- Controls (standard)

Patients (deviant)

Patients (standard)

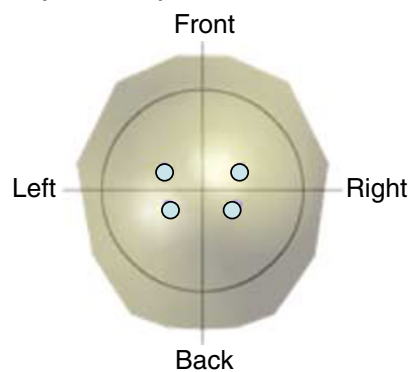

b GFP waveforms (Baseline)
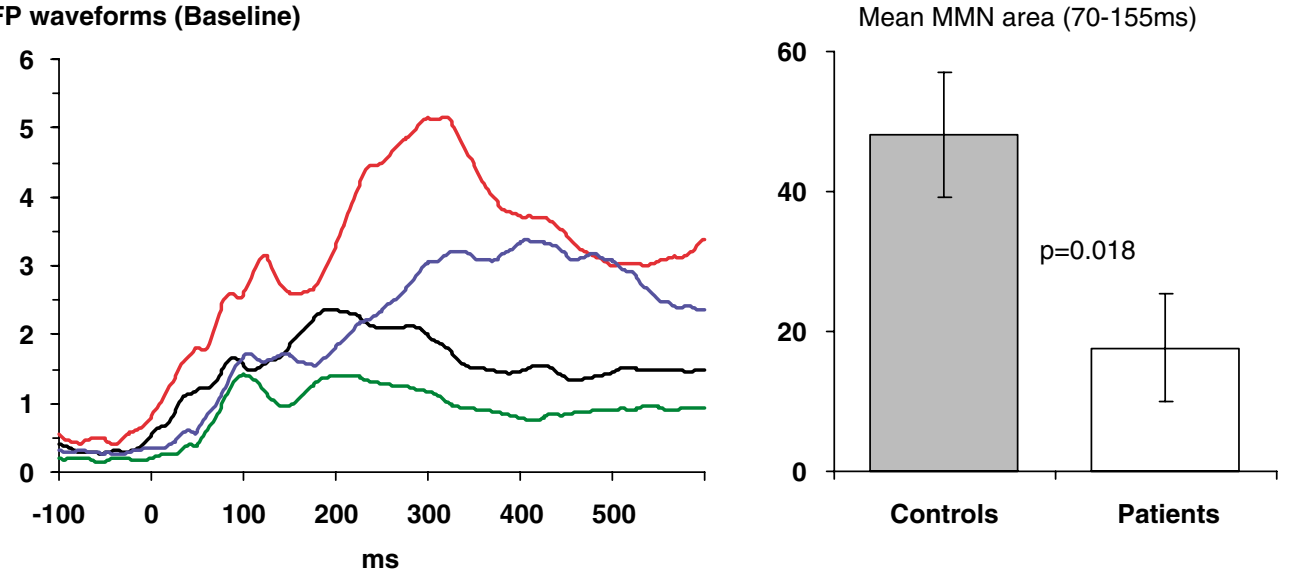

Figure I Electrophysiological responses at protocol onset from schizophrenia patients and healthy controls. (a) The upper panels display exemplar AEP waveforms (voltage as a function of time) from a selection of fronto-central scalp locations (see inset for precise locations) in response to standard and deviant stimuli in both populations (color scheme indicated). Of particular interest is the initial negative-going component when an MMN is typically observed in healthy controls. The bar graph illustrates the group-averaged MMN ( \pm SEM) during the 70-155 ms post-stimulus period and averaged across the above scalp locations. (b) GFP waveforms from all stimulus conditions and MMN magnitude are displayed for schizophrenia patients and healthy controls. Conventions are identical to those in (a). 
diagnosis for schizophrenia as the between-subject factors and stimulus condition and electrode as within-subject factors. Importantly, there was a significant interaction between diagnosis for schizophrenia and stimulus condition $\left(\mathrm{F}_{(1,20)}=11.605 ; p=0.003 ; \eta_{\mathrm{p}}^{2}=0.367\right)$. Given this interaction, we then quantified the MMN for each population by calculating the average difference between responses to standard and deviant stimulus conditions across the four electrodes (bar graph in Figure 1a). These values were then submitted to a one-way ANOVA using diagnosis for schizophrenia as the between-subject factors. As expected, the MMN amplitude was significantly smaller in patients than in controls $\left(\mathrm{F}_{(1,20)}=11.626 ; p=0.003 ; \eta_{\mathrm{p}}^{2}=0.368\right)$. An identical pattern of results was evident in the GFP waveforms (Figure $1 \mathrm{~b}$ ), which represent a global measure of the AEPs across the entire electrode montage and therefore are not influenced by a pre-selection of specific electrodes for analyses. GFP area measures ( $v s$ the $0 \mu \mathrm{V}$ baseline) over the $70-155 \mathrm{~ms}$ interval were submitted to a $2 \times 2$ repeated measures ANOVA, using diagnosis for schizophrenia as the between-subject factors and stimulus condition as the within-subject factors. As above, there was a significant interaction between diagnosis for schizophrenia and stimulus condition $\left(\mathrm{F}_{(1,20)}=6.629 ; p=0.018 ; \eta_{\mathrm{p}}^{2}=0.249\right)$, a significant main effect of diagnosis for schizophrenia $\left(\mathrm{F}_{(1,20)}=10.865 ; p=0.004 ; \eta_{\mathrm{p}}^{2}=0.352\right)$, as well as a significant main effect of stimulus condition $\left(\mathrm{F}_{(1,20)}=30.914\right.$; $\left.p<0.001 ; \eta_{\mathrm{p}}^{2}=0.607\right)$. We then assessed whether the MMN from each group was larger than the $0 \mu \mathrm{V}$ baseline (see bar graphs in Figure 1b). For controls, the mean MMN measured with GFP was $48.0 \pm 8.9 \mu \mathrm{V}$ and was significantly larger than baseline $\left(t_{(10)}=5.387 ; p<0.001\right)$. For patients, the mean MMN measured with GFP was $17.6 \pm 7.7 \mu \mathrm{V}$ and was significantly larger than the $0 \mu \mathrm{V}$ baseline $\left(t_{(10)}=2.276\right.$; $p=0.046$ ). Thus, analyses both at the level of single electrodes and also at the level of the global electric field strength confirmed that the MMN was deficient in our group of patients at protocol onset.

We also examined whether the later P300 component was impaired in patients at the time of baseline measurements. For this analysis, GFP area measures were calculated over the $236-600 \mathrm{~ms}$ interval (ie the same time period used below for the contrast of measurements during NAC and placebo treatments). These values were submitted to a $2 \times 2$ ANOVA as above. Only the main effect of stimulus condition was significant $\left(\mathrm{F}_{(1,20)}=50.325 ; p<0.001 ; \eta_{\mathrm{p}}^{2}=0.716\right)$, whereas the main effect of diagnosis and the interaction between these factors both failed to meet the 0.05 significance criterion (all $p$-values $>0.05$ ). This pattern of results would suggest that the P300 was intact in patients at protocol onset.

However, to reduce the risk of Type II errors (given the large time window identified for the P300 component), we also analyzed the GFP area over smaller time intervals $(236-300,302-400,402-500$, and 502-600 ms) with the same $2 \times 2$ design described above. Over the $236-300 \mathrm{~ms}$ interval, there were main effects of stimulus condition $\left(\mathrm{F}_{(1,20)}=\right.$ $\left.77.859 ; p<0.001 ; \eta_{\mathrm{p}}^{2}=0.796\right)$ and group $\left(\mathrm{F}_{(1,20)}=9.950\right.$; $\left.p=0.005 ; \eta_{\mathrm{p}}^{2}=0.332\right)$ as well as a significant interaction $\left(\mathrm{F}_{(1,20)}=8.315 ; p=0.009 ; \eta_{\mathrm{p}}^{2}=0.294\right)$. Over all of the ensuing periods (ie $302-400,402-500$, and $502-600 \mathrm{~ms}$ ) there was only a main effect of stimulus condition (all $p$-values $<0.001)$. For none of these time windows was there either a main effect of group or an interaction between group and stimulus condition.

\section{Treatment Efficacy: NAC vs Placebo}

Behavioral results. Reaction times on the auditory discrimination task did not differ between patients following NAC (mean \pm SEM $=800 \pm 17 \mathrm{~ms}$ ) and placebo treatment $(829 \pm 30 \mathrm{~ms})$. Likewise, performance accuracy was near ceiling levels for both treatments (NAC: $100 \pm 0 \%$; placebo: $97 \pm 3 \%)$. This pattern of results thus argues against differences in selective attention or task performance as underlying AEP differences between treatments.

Electrophysiological results. As was the case at protocol onset, the topographic pattern analysis identified the same sequence of maps in response to both treatments and stimulus conditions. Thus, there was no evidence for differences in the configuration of underlying brain networks with NAC treatment or between stimulus conditions. The results of this analysis will therefore not be discussed further. The N1 component of the AEP was identified over the $70-155 \mathrm{~ms}$ period, and the sequence of group-average AEP response topographies over the $80-120 \mathrm{~ms}$ interval are displayed in Figure 2 for each treatment and stimulus condition. A fronto-central negativity was observed across both NAC and placebo treatments and both stimulus conditions. Inspection of the topography of the difference between stimulus conditions suggests that there was a robust MMN following NAC but not placebo treatment. To facilitate comparison of the present results with those of prior studies, we analyzed AEP difference waves recorded from midline scalp sites $\mathrm{Fz}$ and $\mathrm{Pz}$ as a function of treatment type (Figure 2c). AEP difference waves recorded at $\mathrm{Fz}$ show that there was a reliable MMN following NAC treatment, but not following placebo treatment. Results of millisecond-by-millisecond paired $t$-tests between standard and deviant AEPs for each treatment type are shown below the difference waveforms.

AEP waveforms from four fronto-central electrodes, selected based on the above topographic maps, are displayed in Figure 3a and suggest that N1 responses to deviant sounds were stronger following NAC than placebo treatment (red and blue traces, respectively), whereas responses to standard sounds did not differ (black and green traces, respectively). Area measures ( $v s$ the $0 \mu \mathrm{V}$ baseline) were taken from these electrodes over the $70-155 \mathrm{~ms}$ interval and submitted to a $2 \times 2 \times 4$ repeated measures ANOVA, using treatment, stimulus condition, and electrode as the within-subject factors. The main effect of treatment did not reach the 0.05 significance criterion $\left(\mathrm{F}_{(1,6)}=0.739 ; p=0.423 ; \eta_{\mathrm{p}}^{2}=0.110\right)$, providing no evidence for a global change in AEP amplitude with NAC treatment. There was a significant main effect of stimulus condition $\left(\mathrm{F}_{(1,6)}=21.206 ; p=0.004 ; \eta_{\mathrm{p}}^{2}=0.779\right)$, following from the generally stronger responses to deviant than to standard stimulus conditions. Most critically, there was a significant interaction between treatment and stimulus condition $\left(\mathrm{F}_{(1,6)}=8.799 ; p=0.025 ; \eta_{\mathrm{p}}^{2}=0.595\right)$. Given this interaction, we then quantified the MMN for each treatment by calculating the average difference between responses to 
a AEP topography: NAC treatment

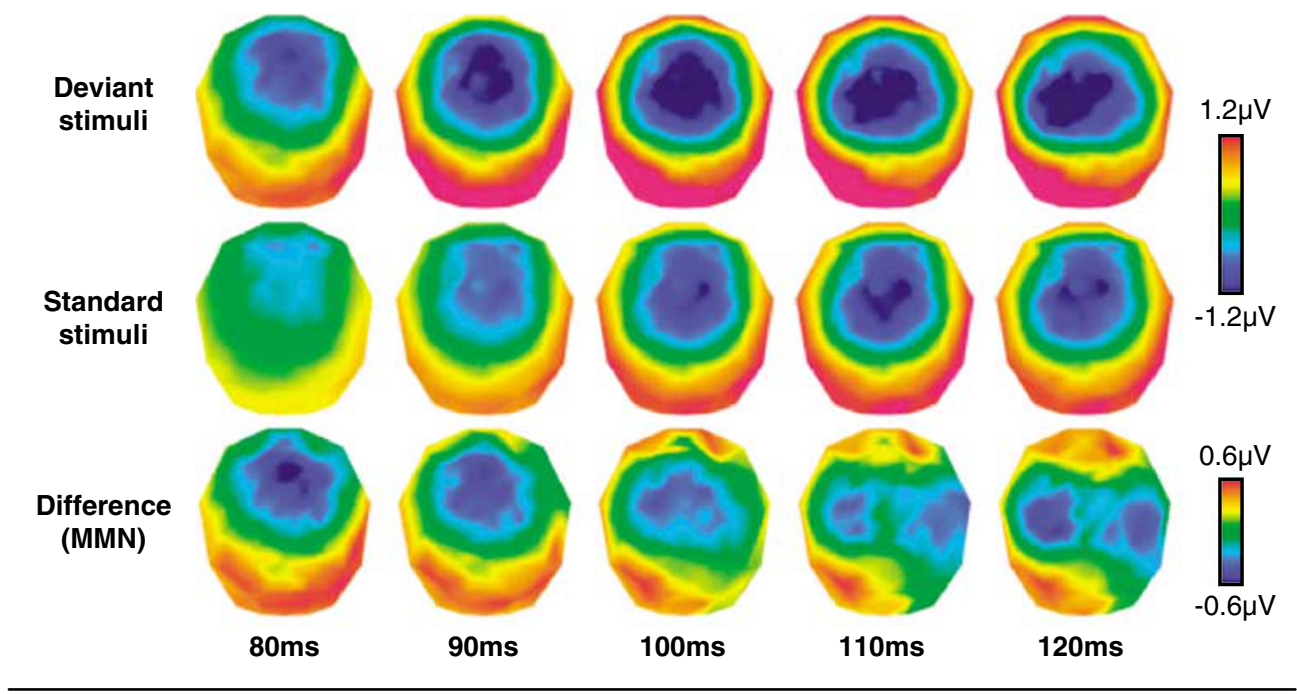

b AEP topography: Placebo treatment

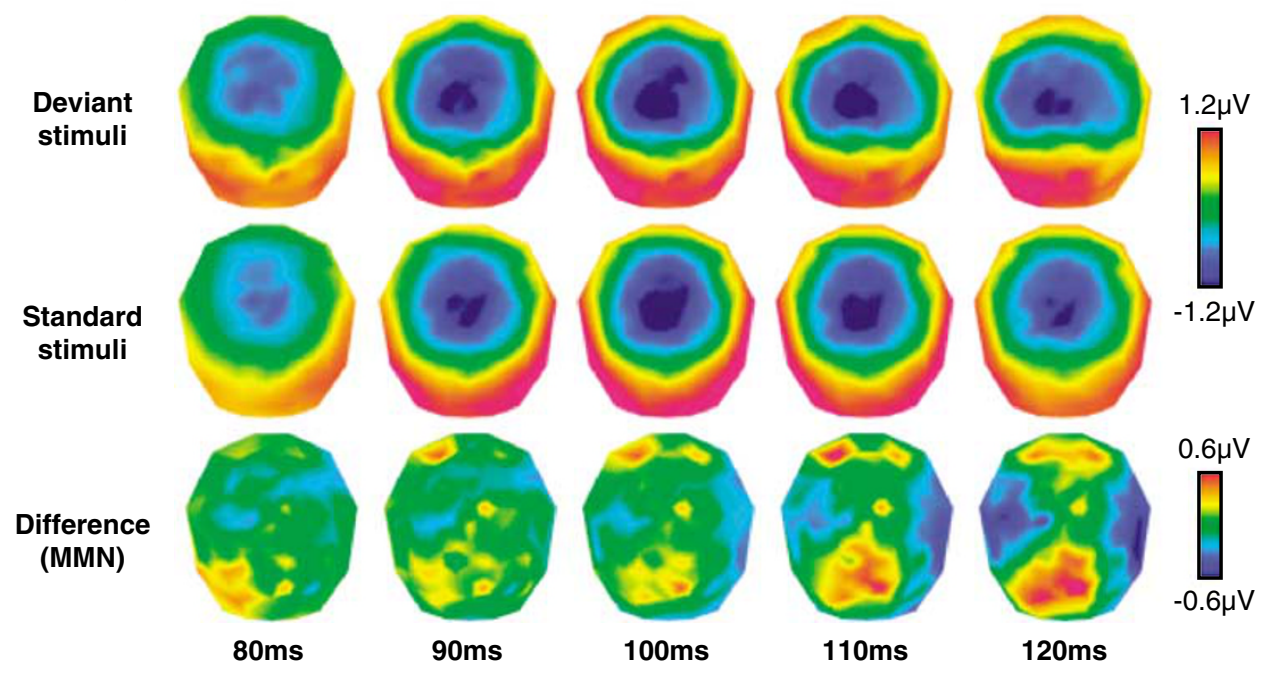

C Target-Distracter difference waveforms
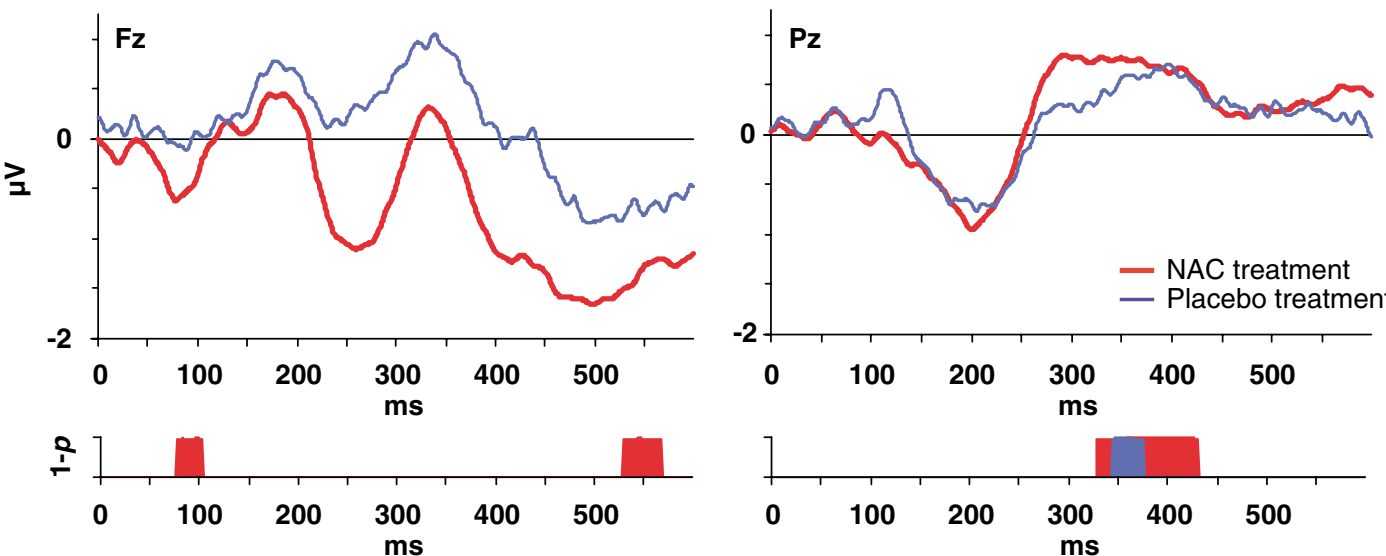

Figure 2 Topographic maps of AEP responses showing the distribution of voltage amplitude across the scalp at $10 \mathrm{~ms}$ intervals from 80 to $120 \mathrm{~ms}$ poststimulus onset. Panels ( $a$ and b) show responses following NAC and placebo treatment, respectively, for each stimulus condition as well as their difference (ie the MMN). (c) Depicts different waveforms for each type of treatment as recorded at frontal and parietal midline sites (Fz and Pz, respectively) as well as the results of millisecond-by-millisecond paired $t$-tests between responses to deviants and standards ( $\mathrm{I}-\mathrm{p}$ plotted as a function of time). 

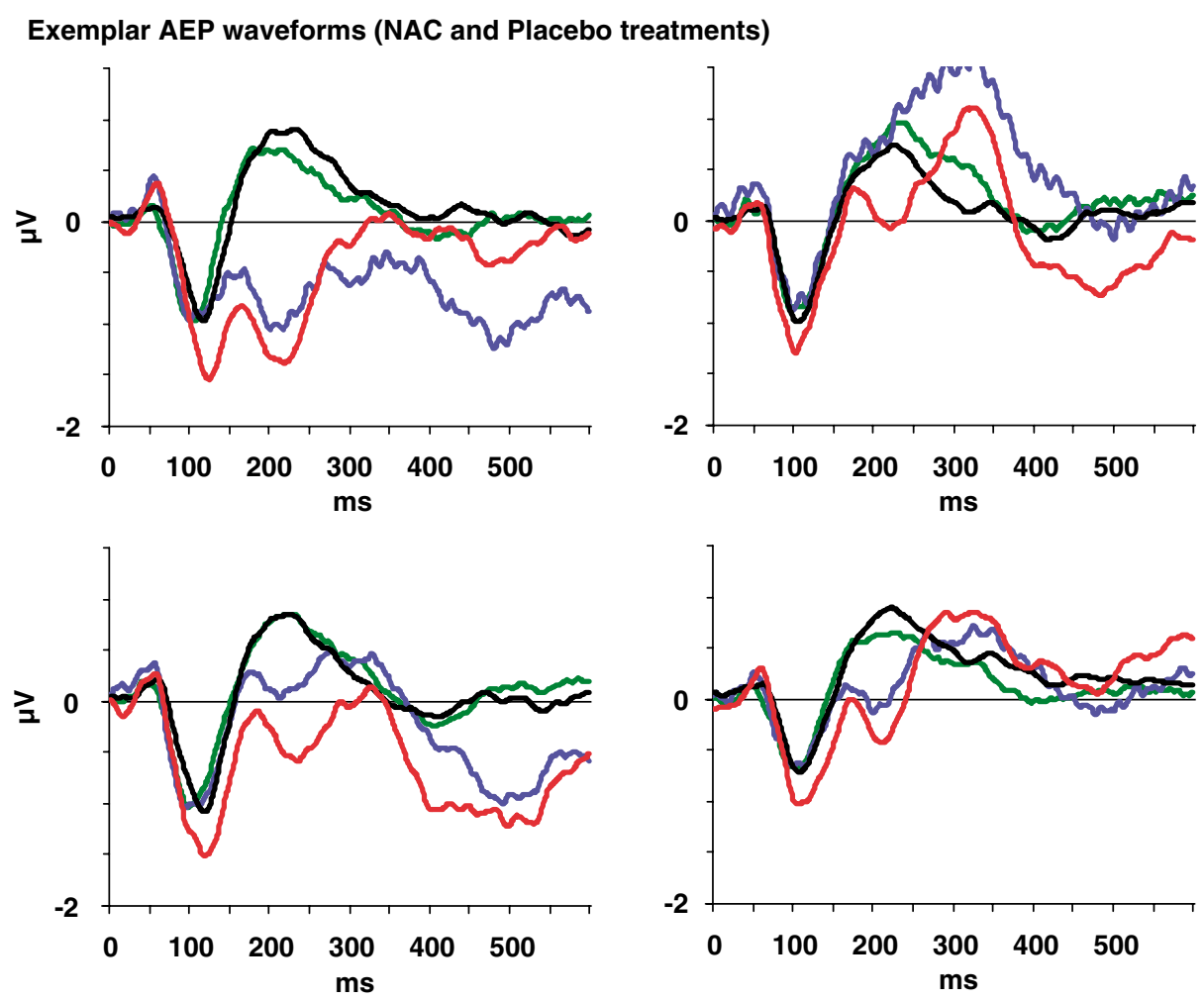

Mean MMN area (70-155ms) across electrodes
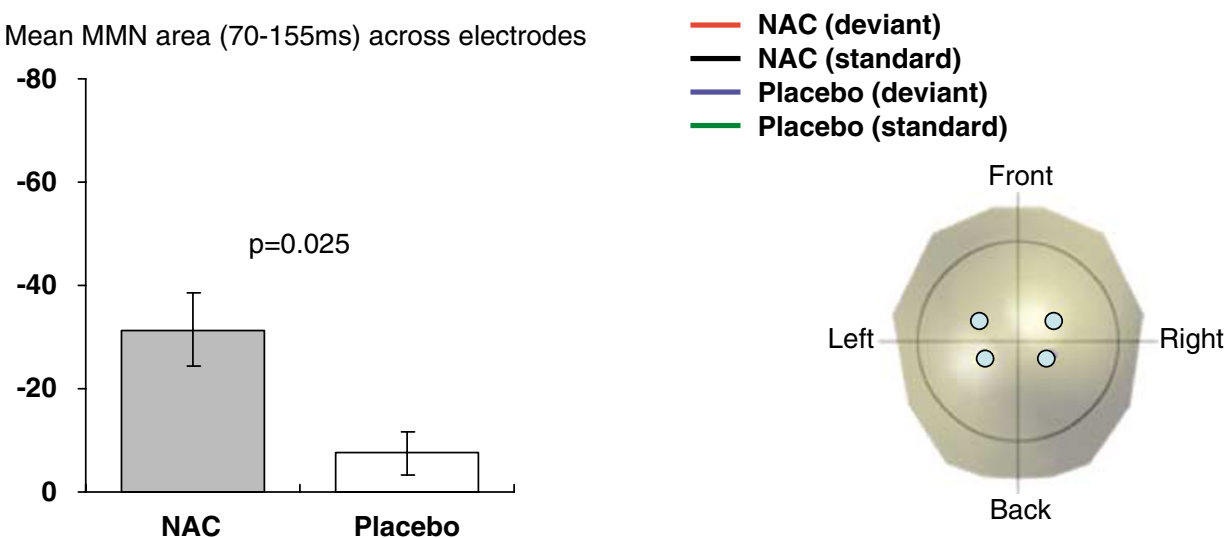

Figure 3 Electrophysiological responses at exemplar scalp sites following NAC and placebo treatment. The upper panels display AEP waveforms (voltage as a function of time) from a selection of fronto-central scalp locations (see inset for precise locations) in response to standard and deviant stimuli after each type of treatment (color scheme indicated). The bar graph illustrates the group-averaged MMN ( \pm SEM) during the 70-I55 ms post-stimulus period and averaged across the above scalp locations.

standard and deviant stimulus conditions across the four electrodes (bar graph in Figure 3a). These values were then tested with a paired $t$-test and were significantly larger following NAC than placebo $(-31.3 \pm 7.1 v s-7.5 \pm 4.2 \mu \mathrm{V}$, respectively; $\left.t_{(6)}=2.966 ; p=0.025\right)$. Of particular note is that MMN responses following placebo did not significantly differ from zero $\left(t_{(6)}=1.789 ; p=0.124\right)$, whereas those following NAC $\operatorname{did}\left(t_{(6)}=4.428 ; p=0.004\right.$; see also Figure $2 \mathrm{c}$ for difference waveforms at electrode $\mathrm{Fz}$ ). This pattern would suggest that the above significant interaction follows from the emergence of an MMN following NAC treatment, which in the present paradigm is likely to be temporally superimposed on the $\mathrm{N} 1$ component of the AEP.

An identical pattern of results was evident in the GFP waveforms (Figure 4a). GFP area measures ( $v$ s the $0 \mu \mathrm{V}$ baseline) over the $70-155 \mathrm{~ms}$ interval were submitted to a $2 \times 2$ repeated measures ANOVA, using treatment and stimulus condition as the within-subject factors. The main effect of treatment did not reach the 0.05 significance criterion $\left(\mathrm{F}_{(1,6)}=0.081 ; p=0.785 ; \eta_{\mathrm{p}}^{2}=0.013\right)$, providing no evidence for a global change in GFP amplitude with NAC treatment and also arguing against a general GFP modulation as a function of time (ie the majority of patients received NAC and then placebo). As above, there was a significant main effect of stimulus condition $\left(\mathrm{F}_{(1,6)}=\right.$ 20.562; $\left.p=0.004 ; \eta_{\mathrm{p}}^{2}=0.774\right)$ and a significant interaction between treatment and stimulus condition $\left(\mathrm{F}_{(1,6)}=6.141\right.$; $\left.p<0.05 ; \eta_{\mathrm{p}}^{2}=0.506\right)$. We then directly analyzed MMN amplitude in two ways. The first was with a paired $t$-test, which revealed a significantly larger MMN following NAC 

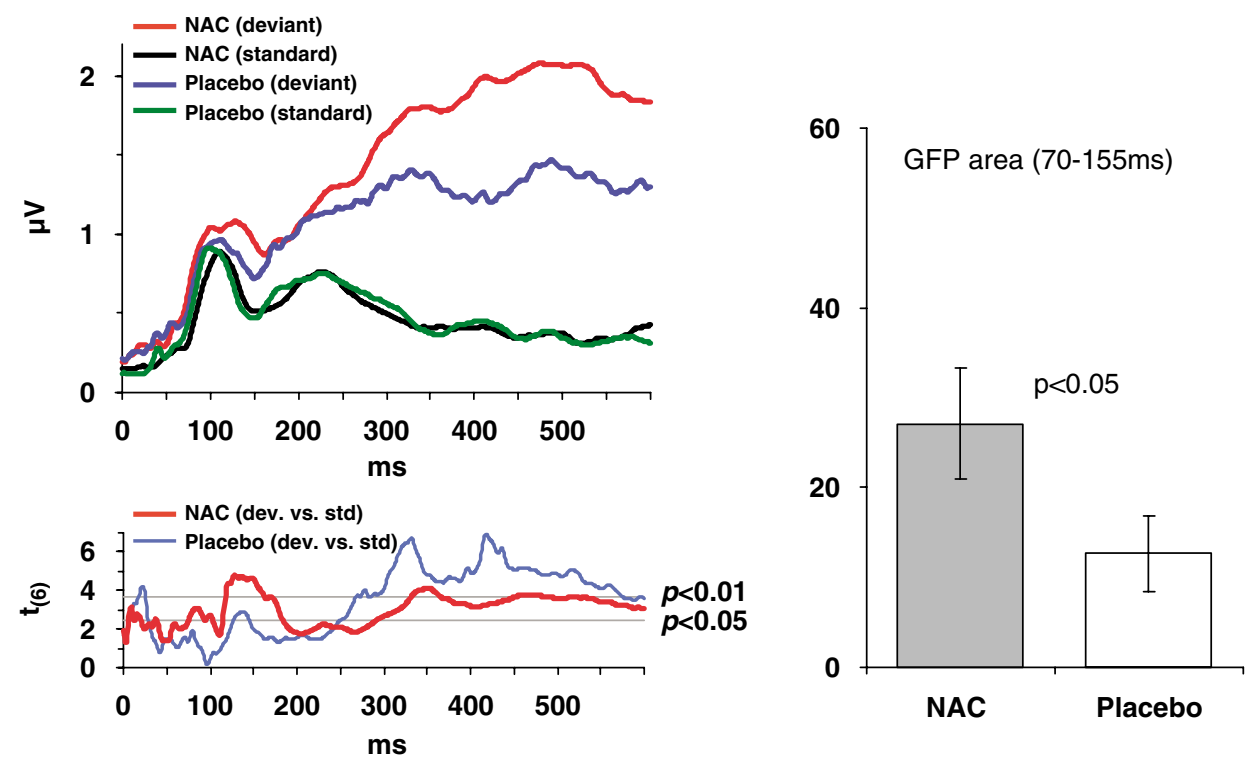

b Group-Average Source Estimations (70-155ms)

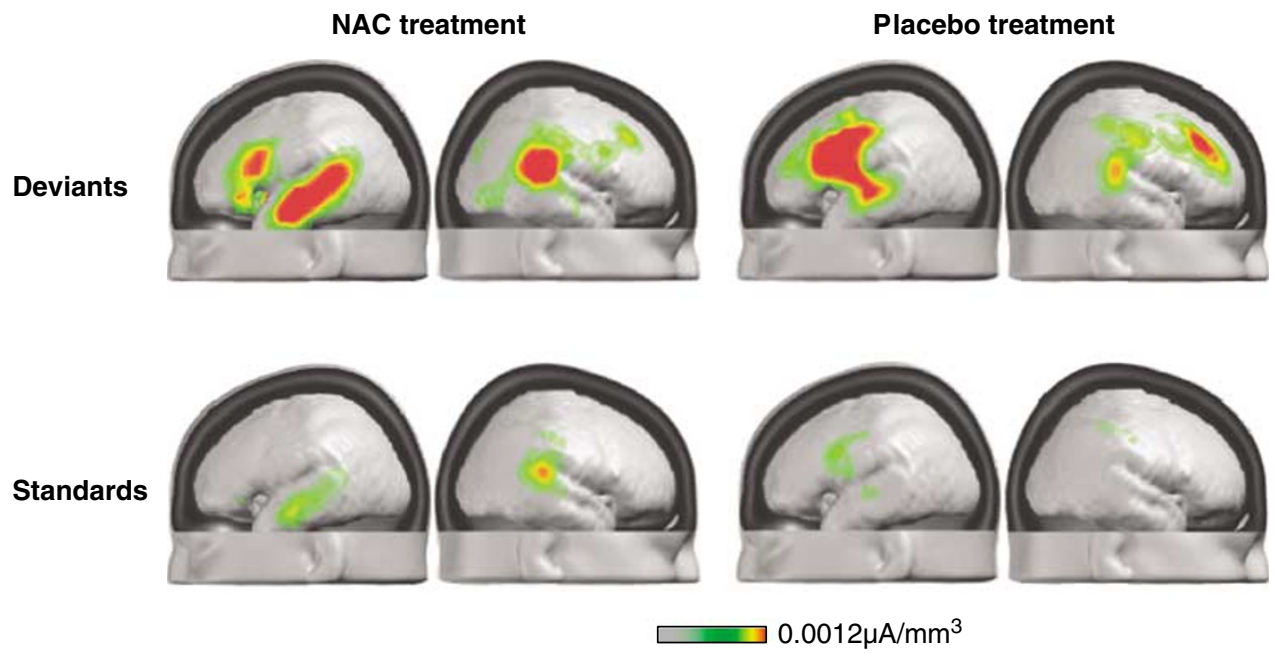

Figure 4 Electrophysiological responses and source estimations following NAC and placebo treatment. (a) GFP waveforms from all stimulus conditions are displayed following each type of treatment along with millisecond-by-millisecond contrasts between responses to deviants and standards as well as MMN magnitude (area over the 70-155 ms period). Conventions are identical to those in Figure 3. (b) Group-averaged source estimations following NAC treatment are shown separately for responses to deviant and standard sound stimuli. Stronger sources for deviant sounds are evident within superior temporal cortices and frontal cortices, consistent with enhanced MMN generation (see Results section for details).

than placebo $(27.1 \pm 6.1$ vs $12.7 \pm 4.2 \mu \mathrm{V}$, respectively, $t_{(6)}=2.458 ; p<0.05$; bar graphs in Figure $4 \mathrm{a}$ ), although in both cases the MMN measured using GFP was significantly greater than zero ( $p$-values $<0.025)$. The second was with a millisecond-by-millisecond paired $t$-test between responses to deviants and standards during each type of treatment, separately (see Figure 4a). This analysis shows that a significant differential response (ie MMN) was observed following NAC treatment over the $\sim 70-200$ ms period that was diminished in the case of placebo treatment. Thus, analyses at the level of single electrodes and also at the level of the global electric field strength confirmed that the MMN was improved following NAC but not placebo treatment. We would further note that all but one of the patients exhibited a qualitatively larger MMN while receiving NAC relative to when receiving placebo treatment (see Supplementary Figure S2).

We also examined whether the later P300 component was modulated by whether or not patients were receiving NAC or placebo. For this analysis GFP area measures were calculated over the $235-600 \mathrm{~ms}$ interval (see waveforms in Figure 4), using the above-mentioned topographic pattern analysis as a basis for the identification of AEP components. These values were submitted to a $2 \times 2$ ANOVA using treatment and stimulus condition as within-subject factors. Only the main effect of stimulus condition was significant $\left(\mathrm{F}_{(1,6)}=30.212 ; p=0.002 ; \eta_{\mathrm{p}}^{2}=0.834\right)$, whereas the main effect of treatment and the interaction between these factors 
both failed to meet the 0.05 significance criterion (all $p$-values $>0.30$ ). This pattern of results would suggest that the P300 was intact in patients both when receiving placebo as well as when receiving NAC. This AEP component showed no indication that it was modified by NAC treatment, in contrast to the earlier MMN response. This pattern of results is furthermore consistent with that observed in the recordings prior to protocol onset (see Figure 1). In order to reduce the risk of Type II errors (given the large time window identified for the P300 component), we also analyzed the GFP area over smaller time intervals (235-300, 301-400, 401-500, and 501-600 ms) with the same $2 \times 2$ design described above. For each of these time intervals, there was a significant main effect of stimulus condition (all F-values $>12.669 ; p$-values $<0.012$ ). For no time interval was there either a significant main effect of treatment (all F-values $<0.701$; $p$-values $>0.435$ ) or a significant interaction between factors (all F-values $<1.806$; $p$-values $>0.228$ ). Bar graphs illustrating these area measures as well as the topography of the P300 can be found online as Supplementary material (Supplementary Figure S1).

Finally, we estimated the intracranial sources that were active over the $70-155 \mathrm{~ms}$ post-stimulus period for both treatment types and stimulus conditions (Figure $4 \mathrm{~b}$ ). These estimations provide a visualization of the likely underlying generators. Responses to deviant stimuli during NAC treatment resulted in stronger activity within the superior temporal cortex bilaterally relative to all other conditions, whereas activity within frontal cortices was robust in response to deviant stimuli irrespective of treatment type.

No improvement on the psychopathological scales (GCI and PANSS) was observed in our cohort of patients after NAC treatment. However, our data were pooled with those of the larger clinical trial and are presented elsewhere (Berk et al, 2007, unpublished work).

Biochemical results. Levels of thiols linked to GSH were measured in blood from patients after NAC and after placebo treatment. Results are summarized in Table 2. After NAC treatment, an increase in total GSH levels was observed in whole blood $\left(\mathrm{F}_{(1,9)}=20.89 ; p=0.006\right)$ as well as in blood cells $\left(\mathrm{F}_{(1,9)}=29.87 ; p=0.001\right)$. However, this increase relative to placebo did not exceed $10 \%$. There was no increase in cysteine and cysteinyl-glycine. No correlation was found between the magnitude of these biochemical changes and our electrophysiological measures.

\section{DISCUSSION}

We show that administration of NAC, a GSH precursor, to schizophrenia patients resulted in improved auditory cortical functioning as indexed by the MMN. There is increasing evidence that the pathophysiology of schizophrenia entails both high-level cognitive impairments as well as (and perhaps as a consequence of low-level sensory processing deficits across sensory modalities (Butler and Javitt, 2005; Foxe et al, 2005; Javitt et al, 1999). For example, previous research has consistently shown that the MMN is impaired in schizophrenia patients (Catts et al, 1995; Javitt et al, 1993, 1998; Shelley et al, 1991; Shutara et al, 1996; for review see Turetsky et al, 2007), and this was again the case
Table 2 Levels of GSH and its Cysteine-Related Precursors Measured in Blood from Patients after NAC Treatment and Placebo

\begin{tabular}{lccc}
\hline & $\begin{array}{c}\text { NAC treatment } \\
\text { (mean } \pm \text { SEM) }\end{array}$ & $\begin{array}{c}\text { Placebo } \\
\text { (mean } \pm \text { SEM) }\end{array}$ & $\begin{array}{c}\text { ANOVA } \\
\text { P-value }\end{array}$ \\
\hline $\begin{array}{l}\text { GSH in blood cells } \\
(\mu \mathrm{mol} / \mathrm{ml} \text { blood) }\end{array}$ & $0.82 \pm 0.05$ & $0.76 \pm 0.06$ & $\mathbf{0 . 0 0 1}$ \\
$\begin{array}{l}\mathrm{GSH} \text { in whole blood } \\
(\mu \mathrm{mol} / \mathrm{ml} \text { blood })\end{array}$ & $0.89 \pm 0.06$ & $0.81 \pm 0.07$ & $\mathbf{0 . 0 0 6}$ \\
$\begin{array}{l}\text { Cysteine in plasma } \\
(\mu \mathrm{mol} / \mathrm{l})\end{array}$ & $248.0 \pm 10.0$ & $262.4 \pm 8.3$ & 0.20 \\
$\begin{array}{l}\text { Cysteinyl-glycine in } \\
\text { plasma }(\mu \mathrm{mol} / \mathrm{l})\end{array}$ & $43.1 \pm 3.1$ & $46.5 \pm 7.3$ & 0.17 \\
\hline
\end{tabular}

Bold $P$-values indicate significant difference.

for our patients at protocol onset (Figure 2). Interestingly, the greater the MMN impairment, the lower are the Global Assessment of Functioning Scale ratings, suggesting that MMN is linked to global impairments in everyday functioning in schizophrenia patients (Kawakubo and Kasai, 2006; Light and Braff, 2005a, b). MMN generation is thought to rely on intact NMDA receptor function. We propose that NAC is leading to an improvement in NMDA receptor function that is non-invasively detectable as modification in MMN generation. In the following, we first discuss the AEP effects and then putative mechanisms of action whereby NAC could produce such effects.

At protocol onset, patients exhibited deficient MMN generation (Figure 2), despite their ability to perform the oddball discrimination task with equal accuracy and speed as control subjects. Additionally, there was no evidence that AEP amplitude in general was impaired between patients and controls. Our results would instead indicate that it was specifically MMN generation that was impaired - that is the mechanism mediating the comparison between standard and deviant stimuli. This pattern of results is in solid agreement with those obtained following the infusion of the NMDA antagonist phencyclidine in non-human primates (Javitt et al, 1996) and administration of ketamine to healthy human volunteers (Umbricht et al, 2000). In these studies, MMN was reduced, despite intact sensory responses. It is therefore unlikely that the MMN deficit observed at protocol onset in the present study follows from a general impairment in auditory sensory processing or is a consequence of attentional impairments in patients; a conclusion supported as well by patients' intact performance and $\mathrm{P} 300$ responses.

The principal finding of our study is that administration of NAC improved MMN generation (Figures 2-4). On average, there was a four-fold and two-fold enhancement of MMN amplitude, as measured from AEP and GFP waveforms, respectively. There was no evidence of a general modification of AEP responses nor was there evidence of performance changes on the task. Reaction times and accuracy did not differ across treatments. It is further unlikely that the present results reflect learning-induced plasticity, because the majority of patients (five of the seven) first received NAC and then placebo during the course of the protocol (Figure 1). If such were the case, a 
larger MMN would be predicted for the EEG recording at the end of the protocol (eg Spierer et al, 2007 for a recent example in healthy participants), which in our case was most often after placebo rather than NAC treatment. To further exclude the possibility that treatment order was mediating our effects, we re-analyzed the GFP data including order as a between-subject factors. As before, there was a main effect of stimulus condition $\left(\mathrm{F}_{(1,5)}=\right.$ $\left.17.221 ; p=0.009 ; \eta_{\mathrm{p}}^{2}=0.775\right)$ as well as a significant interaction between treatment and stimulus condition $\left(\mathrm{F}_{(1,5)}=7.326 ; p=0.042 ; \eta_{\mathrm{p}}^{2}=0.594\right)$. However, treatment order did not result in a significant main effect or interaction with either within-subject factors.

An increase of GSH levels in blood was measured in our patients after NAC treatment (Table 2), showing that NAC was absorbed, at least in part, by the gastrointestinal system. No increase in cysteine levels was observed in blood from patients after NAC treatment. This could suggest that all the deacetylated cysteine is immediately used and that higher doses of NAC could be considered for treatment. On the other hand, even though single oral doses of NAC leads to an increase in NAC levels in plasma, it does not appear to accumulate (Borgstrom and Kagedal, 1990). In this case, a more frequent intake of NAC would ensure more sustained concentrations.

We hypothesize that a fraction of the oral NAC entered blood flow, crossed the blood-brain barrier (Farr et al, 2003), was deacetylated into cysteine that could in turn be used to increase GSH levels. GSH being the major antioxidant in a cell system, its presence is necessary to maintain equilibrium on the redox status. In fact, redox-sensitive proteins such as NMDA receptors can have their activity decreased when the ratio between GSH and its oxidized form becomes too low (Janaky et al, 1993; Sucher and Lipton, 1991). Moreover, GSH deficit in hippocampus slices leads to NMDA receptor hypofunction and inhibition of long-term potentiation (Steullet et al, 2006). In patients with low brain GSH, NAC could increase GSH levels, restoring normal GSH levels and thus improving NMDA reception functioning, which is thought to be reflected by the amplitude of the MMN (Javitt et al, 1996). Therefore, the present observation of an increase in MMN amplitude after NAC treatment is likely to reflect improved NMDA receptor function.

However, from our data, we cannot unequivocally conclude that the effect of NAC is via its cysteine donor property. In fact, NAC being an antioxidant itself, it is possible that it has a direct effect on the redox properties of NMDA receptors. NAC can also affect gene regulation of detoxifying enzymes by activating redox-sensitive transcription factors, such as nuclear factor $\kappa \mathrm{B}$ and activator protein 1 (Cotgreave, 1997). To assess whether NAC elevates GSH levels in the brain of schizophrenia patients, in vivo magnetic resonance spectroscopy (MRS) would be necessary. However, it has been reported that with current MRS methods, the signal-to-noise ratio would need to be improved to observe discrete increases in brain GSH levels (Trabesinger et al, 1999).

Considering that brain GSH deficiency in schizophrenia seems to be associated with a defect in the key synthesizing enzyme glutamyl-cysteine ligase (Gysin et al, 2007; Tosic et al, 2006), that conjugates cysteine to glutamate in the first step of GSH synthesis, a cysteine precursor might not represent the best substance to use to increase GSH levels. Substances that bypass this enzyme would represent better choices; however, raising new challenges for their efficient delivery across the blood-brain barrier.

The present study was conducted in the context of a multicenter clinical trial. Our center conducted a double-blinded and crossover study, wherein NAC was given as an add-on treatment ( $2 \mathrm{~g} /$ day for 2 months). No significant changes in the psychopathology was observed in our cohort of patients, which may be due to the relatively small sample size. Nonetheless, the effectiveness of NAC in reducing clinical severity was demonstrated over the whole set of 140 patients included in the multi-center double-blinded trial (Berk et al, 2007, unpublished work), which included data from our group of patients. An improvement on the Clinical Global Impression was observed 2 weeks after the beginning of the trial and was sustained throughout the 6-month trial period. An improvement of negative symptoms on the Positive and Negative Symptoms Scale was also significant after 6 months of treatment with NAC. These findings suggest that longer treatments and larger sample sizes may be required to observe behavioral improvements following NAC treatment, whereas the present electrophysiological effects were nonetheless reliable after a relatively short treatment duration and highlight the clinical utility of brain imaging methods. We would emphasize that although we have sufficient power to obtain statistically reliable positive results with only seven patients, confirming our results with a larger sample size will be necessary. A new and larger study of first-episode psychosis patients is currently being initiated to further investigate the results supported by the present study as well as whether an earlier intervention will be more efficient. An additional consideration in the present study is its use of a large frequency difference (ie $1000 \mathrm{~Hz}$ ) between distracters and targets as well as an active discrimination paradigm to elicit both MMN and P300 responses. Some might consider this paradigm as suboptimal in the present investigation. We would note, however, that the MMN is indeed reliably elicited under both active and passive listening conditions (eg Näätänen et al, 1978; Ritter et $a l, 1992)$ and that later components such as N200/P300 are only observed during active paradigms and furthermore may not be a pure index of sensory discrimination abilities (Hillyard et al, 1971). Such things being said, it will clearly be worthwhile for future investigations to examine the MMN under passive conditions as well as when target stimuli are either closer to the deviant's frequency and/or vary in their duration; the latter being a well-established means of dissociating effects specific to the $\mathrm{N} 1$ component from those specific to MMN generation.

In summary, MMN, a component of the AEP that is typically impaired in schizophrenia, was improved following a treatment with NAC, a precursor of GSH, supporting the hypothesis of a deficit in GSH in the brain of schizophrenia patients. Modulating GSH levels in the brain of patients opens new promising approaches for effective therapeutic strategies in schizophrenia.

\section{ABBREVIATIONS}

AEP, auditory evoked potential; EEG, electroencephalograph; GSH, glutathione; NAC, $N$-acetyl-cysteine; MMN, mismatch negativity; NMDA, $N$-methyl-D-aspartate. 


\section{ACKNOWLEDGEMENTS}

We thank Pierre-Georges Meister for the conditioning of the medication and Philippe Maeder for his helpful comments throughout this study. Cartool software (http://brainmapping.unige.ch/Cartool.htm) has been programmed by Denis Brunet, from the Functional Brain Mapping Laboratory, Geneva, Switzerland, and is supported by the Center for Biomedical Imaging (www.cibm.ch) of Geneva and Lausanne. Rolando Grave de Peralta Menendez and Sara Gonzalez Andino developed the LAURA source estimation methods applied here, and Christoph Michel provided additional analysis software. We would also like to acknowledge all the patients and controls who kindly accepted to participate in the study. This work was supported by the Loterie Romande and the Fonds pour la Recherche en Santé du Québec (SL). The Stanley Medical Research Institute supported the manufacture of the clinical trial medication.

\section{DISCLOSURE/CONFLICT OF INTEREST}

Michael Berk declares that over the past three years, he has received compensation from Stanley Medical Research Foundation, MBF, NHMRC, Beyond Blue, Geelong Medical Research Foundation, Bristol Myers Squibb, Eli Lilly, Glaxo SmithKline, Organon, Norvatis, Mayne Pharma and Servier.

The other authors declare that, except for income received from their primary employer, no financial support or compensation has been received from any individual or corporate entity over the past three years for research or professional service and there are no personal financial holdings that could be perceived as constituting a potential conflict of interest.

\section{REFERENCES}

Aydin S, Ozaras R, Uzun H, Belce A, Uslu E, Tahan V et al (2002). $\mathrm{N}$-acetylcysteine reduced the effect of ethanol on antioxidant system in rat plasma and brain tissue. Tohoku J Exp Med 198: 71-77.

Borgstrom L, Kagedal B (1990). Dose dependent pharmacokinetics of $\mathrm{N}$-acetylcysteine after oral dosing to man. Biopharm Drug Dispos 11: 131-136.

Borgstrom L, Kagedal B, Paulsen O (1986). Pharmacokinetics of $N$-acetylcysteine in man. Eur J Clin Pharmacol 31: 217-222.

Brandeis D, Lehmann D, Michel CM, Mingrone W (1995). Mapping event-related brain potential microstates to sentence endings. Brain Topogr 8: 145-159.

Butler PD, Javitt DC (2005). Early-stage visual processing deficits in schizophrenia. Curr Opin Psychiatry 18: 151-157.

Cabungcal JH, Nicolas D, Kraftsik R, Cuénod M, Do KQ, Hornung JP (2006). Glutathione deficit during development induces anomalies in the rat anterior cingulate GABAergic neurons: relevance to schizophrenia. Neurobiol Dis 22: 624-637.

Cabungcal JH, Preissman D, Delseth C, Cuénod M, Do KQ, Schenk F (2007). Transitory glutathione deficit during brain development induces cognitive impairment in juvenile and adult rats: relevance to schizophrenia. Neurobiol Dis 26: 634-645.

Castagné V, Cuénod M, Do KQ (2004a). An animal model with relevance to schizophrenia: sex-dependent cognitive deficits in osteogenic disorder-Shionogi rats induced by glutathione synthesis and dopamine uptake inhibition during development. Neuroscience 123: 821-834.
Castagné V, Rougemont M, Cuénod M, Do KQ (2004b). Low brain glutathione and ascorbic acid associated with dopamine uptake inhibition during rat's development induce long-term cognitive deficit: relevance to schizophrenia. Neurobiol Dis 15: 93-105.

Catts SV, Shelley AM, Ward PB, Liebert B, McConaghy N, Andrews S et al (1995). Brain potential evidence for an auditory sensory memory deficit in schizophrenia. Am J Psychiatry 152: 213-219.

Choi YB, Lipton SA (2000). Redox modulation of the NMDA receptor. Cell Mol Life Sci 57: 1535-1541.

Cotgreave IA (1997). $\mathrm{N}$-acetylcysteine: pharmacological considerations and experimental and clinical applications. Adv Pharmacol 38: 205-227.

Coyle JT (2006). Glutamate and schizophrenia: beyond the dopamine hypothesis. Cell Mol Neurobiol 26: 365-384.

Do KQ, Bovet P, Cabungcal JH, Conus P, Gysin R, Lavoie S et al Redox dysregulation in schizophrenia: genetic susceptibility and pathophysiological mechanisms. In: Lajtha A (ed). Hankbook of Neurochemistry and Molecular Neurobiology. Springer, London, New York, in press.

Do KQ, Trabesinger AH, Kirsten-Krüger M, Lauer CJ, Dydak U, Hell D et al (2000). Schizophrenia: glutathione deficit in cerebrospinal fluid and prefrontal cortex in vivo. Eur J Neurosci 12: $3721-3728$.

Ercal N, Treeratphan P, Hammond TC, Matthews RH, Grannemann NH, Spitz DR (1996). In vivo indices of oxidative stress in lead-exposed C57BL/6 mice are reduced by treatment with meso-2,3-dimercaptosuccinic acid or N-acetylcysteine. Free Radic Biol Med 21: 157-161.

Farr SA, Poon HF, Dogrukol-Ak D, Drake J, Banks WA, Eyerman E et al (2003). The antioxidants alpha-lipoic acid and $\mathrm{N}$-acetylcysteine reverse memory impairment and brain oxidative stress in aged SAMP8 mice. J Neurochem 84: 1173-1183.

Foxe JJ, Murray MM, Javitt DC (2005). Filling-in in schizophrenia: a high-density electrical mapping and source-analysis investigation of illusory contour processing. Cereb Cortex 15: 1914-1927.

Fu AL, Dong ZH, Sun MJ (2006). Protective effect of $\mathrm{N}$-acetyl-1cysteine on amyloid [beta]-peptide-induced learning and memory deficits in mice. Brain Res 1109: 201-206.

Garey LJ, Ong WY, Patel TS, Kanani M, Davis A, Mortimer AM et al (1998). Reduced dendritic spine density on cerebral cortical pyramidal neurons in schizophrenia. J Neurol Neurosurg Psychiatry 65: 446-453.

Glantz LA, Lewis DA (2000). Decreased dendritic spine density on prefrontal cortical pyramidal neurons in schizophrenia. Arch Gen Psychiatry 57: 65-73.

Grave de Peralta Menendez R, Gonzalez Andino S, Lantz G, Michel CM, Landis T (2001). Noninvasive localization of electromagnetic epileptic activity. I. Method descriptions and simulations. Brain Topography 14: 131-137.

Grave de Peralta Menendez R, Murray MM, Michel CM, Martuzzi R, Gonzalez Andino SL (2004). Electrical neuroimaging based on biophysical constraints. Neuroimage 21: 527-539.

Gysin R, Kraftsik R, Sandell J, Bovet P, Chappuis C, Conus P et al (2007). Impaired glutathione synthesis in schizophrenia convergent genetic and functional evidence. Proc Natl Acad Sci USA 104: 16621-16626.

Hillyard SA, Squires KC, Baver JW, Lindsay PH (1971). Evoked potentials correlates of auditory signal detection. Science 172: 1357-1360.

Jacobsen DW, Gatautis VJ, Green R, Robinson K, Savon SR, Secic M et al (1994). Rapid HPLC determination of total homocysteine and other thiols in serum and plasma: sex differences and correlation with cobalamin and folate concentrations in healthy subjects. Clin Chem 40: 857-858.

Janaky R, Varga V, Saransaari P, Oja SS (1993). Glutathione modulates the $N$-methyl-D-aspartate receptor-activated calcium influx into cultured rat cerebellar granule cells. Neurosci Lett 156: $153-157$. 
Javitt DC, Doneshka P, Zylberman I, Ritter W, Vaughan Jr HG (1993). Impairment of early cortical processing in schizophrenia: an eventrelated potential confirmation study. Biol Psychiatry 33: 513-519.

Javitt DC, Grochowski S, Shelley AM, Ritter W (1998). Impaired mismatch negativity (MMN) generation in schizophrenia as a function of stimulus deviance, probability, and interstimulus/interdeviant interval. Electroencephalogr Clin Neurophysiol 108: 143-153.

Javitt DC, Liederman E, Cienfuegos A, Shelley AM (1999). Panmodal processing imprecision as a basis for dysfunction of transient memory storage systems in schizophrenia. Schizophr Bull 25: 763-775.

Javitt DC, Steinschneider M, Schroeder CE, Arezzo JC (1996). Role of cortical $\mathrm{N}$-methyl-D-aspartate receptors in auditory sensory memory and mismatch negativity generation: implications for schizophrenia. Proc Natl Acad Sci USA 93: 11962-11967.

Javitt DC, Zukin SR (1991). Recent advances in the phencyclidine model of schizophrenia. Am J Psychiatry 148: 1301-1308.

Kamboj A, Kiran R, Sandhir R (2006). Carbofuran-induced neurochemical and neurobehavioral alterations in rats: attenuation by $\mathrm{N}$-acetylcysteine. Exp Brain Res 170: 567-575.

Kawakubo Y, Kasai K (2006). Support for an association between mismatch negativity and social functioning in schizophrenia. Prog Neuropsychopharmacol Biol Psychiatry 30: 1367-1368.

Kohr G, Eckardt S, Luddens H, Monyer H, Seeburg PH (1994). NMDA receptor channels: subunit-specific potentiation by reducing agents. Neuron 12: 1031-1040.

Kolluri N, Sun Z, Sampson AR, Lewis DA (2005). Lamina-specific reductions in dendritic spine density in the prefrontal cortex of subjects with schizophrenia. Am J Psychiatry 162: 1200-1202.

Krijt J, Vackova M, Kozich V (2001). Measurement of homocysteine and other aminothiols in plasma: advantages of using Tris(2-carboxyethyl)phosphine as reductant compared with trin-butylphosphine. Clin Chem 47: 1821-1828.

Krystal JH, Karper LP, Seibyl JP, Freeman GK, Delaney R, Bremner JD et al (1994). Subanesthetic effects of the noncompetitive NMDA antagonist, ketamine, in humans. Psychotomimetic, perceptual, cognitive, and neuroendocrine responses. Arch Gen Psychiatry 51: 199-214.

Lehmann D (1987). Principles of spatial analysis. In: Gevins AS, Rémond A (eds). Methods of Analysis of Brain Electrical and Magnetic Signals. EEG Handbook. Elsevier Science Publishers BV, Amsterdam, pp 309-354.

Lehmann D, Skrandies W (1980). Reference-free identification of components of checkerboard-evoked multichannel potential fields. Electroencephalogr Clin Neurophysiol 48: 609-621.

Light GA, Braff DL (2005b). Mismatch negativity deficits are associated with poor functioning in schizophrenia patients. Arch Gen Psychiatry 62: 127-136.

Light GA, Braff DL (2005a). Stability of mismatch negativity deficits and their relationship to functional impairments in chronic schizophrenia. Am J Psychiatry 162: 1741-1743.

Meister A, Anderson ME (1983). Glutathione. Annu Rev Biochem 52: 711-760.

Meister A, Anderson ME, Hwang O (1986). Intracellular cysteine and glutathione delivery systems. J Am Coll Nutr 5: 137-151.

Michel CM, Murray MM, Lantz G, Gonzalez S, Spinelli L, Grave de Peralta R (2004). EEG source imaging. Clin Neurophysiol 115: 2195-2222.

Murray MM, Imber ML, Savitt DC, Foxe JJ (2006). Boundary completion is automatic and dissociable from shape discrimination. J NSC 26: 12043-12054.

Murray MM, Michel CM, Grave de Peralta R, Ortigue S, Brunet D, Gonzalez Andino S et al (2004). Rapid discrimination of visual and multisensory memories revealed by electrical neuroimaging. Neuroimage 21: 125-135.

Näätänen R, Gaillard AWK, Mantysalo S (1978). Early selectiveattention effect on evoked potential reinterpreted. Acta Psychologica 42: 313-329.

Nurnberger Jr JI, Blehar MC, Kaufmann CA, York-Cooler C, Simpson SG, Harkavy-Friedman J et al (1994). Diagnostic interview for genetic studies. Rationale, unique features, and training. NIMH genetics initiative. Arch Gen Psychiatry 51: 849-859.

Olsson B, Johansson M, Gabrielsson J, Bolme P (1988). Pharmacokinetics and bioavailability of reduced and oxidized $\mathrm{N}$-acetylcysteine. Eur J Clin Pharmacol 34: 77-82.

Perrin F, Pernier J, Bertrand O, Giard MH, Echallier JF (1987). Mapping of scalp potentials by surface spline interpolation. Electroencephalogr Clin Neurophysiol 66: 75-81.

Preisig M, Fenton BT, Matthey ML, Berney A, Ferrero F (1999). Diagnostic interview for genetic studies (DIGS): inter-rater and test-retest reliability of the French version. Eur Arch Psychiatry Clin Neurosci 249: 174-179.

Ritter W, Paavilainen P, Lavikainen J, Reinikainen K, Alho K, Sams M et al (1992). Event-related potentials to repetition and change of auditory stimuli. Electroencephalogr Clin Neurophysiol 83: 306-321.

Robbins TW (2005). Synthesizing schizophrenia: a bottom-up, symptomatic approach. Schizophr Bull 31: 854-864.

Shelley AM, Ward PB, Catts SV, Michie PT, Andrews S, McConaghy N (1991). Mismatch negativity: an index of a preattentive processing deficit in schizophrenia. Biol Psychiatry 30: $1059-1062$.

Shutara Y, Koga Y, Fujita K, Takeuchi H, Mochida M, Takemasa K (1996). An event-related potential study on the impairment of automatic processing of auditory input in schizophrenia. Brain Topogr 8: 285-289.

Spierer L, Tardif E, Sperdin H, Murray MM, Clarke S (2007). Learning-induced plasticity in auditory spatial representations revealed by electrical neuroimaging. J Neurosci 27: 5474-5483.

Steullet P, Neijt HC, Cuénod M, Do KQ (2006). Synaptic plasticity impairment and hypofunction of NMDA receptors induced by glutathione deficit: relevance to schizophrenia. Neuroscience 137: 807-819.

Sucher NJ, Lipton SA (1991). Redox modulatory site of the NMDA receptor-channel complex: regulation by oxidized glutathione. J Neurosci Res 30: 582-591.

Tibshirani R, Walther G, Botstein D, Brown P (2005). Cluster validation by prediction strength. J Comput Graphical Stat 14: 511-528.

Tosic M, Ott J, Barral S, Bovet P, Deppen P, Gheorghita F et al (2006). Schizophrenia and oxidative stress: glutamate cysteine ligase modifier as a susceptibility gene. Am J Hum Genet 79: 586-592.

Trabesinger AH, Weber OM, Duc CO, Boesiger P (1999). Detection of glutathione in the human brain in vivo by means of double quantum coherence filtering. Magn Reson Med 42: 283-289.

Turetsky BI, Calkins ME, Light GA, Olincy A, Radant AD, Swerdlow NR (2007). Neurophysiological endophenotypes of schizophrenia: the viability of selected candidate measures. Schizophr Bull 33: 69-94.

Umbricht D, Schmid L, Koller R, Vollenweider FX, Hell D, Javitt DC (2000). Ketamine-induced deficits in auditory and visual context-dependent processing in healthy volunteers: implications for models of cognitive deficits in schizophrenia. Arch Gen Psychiatry 57: 1139-1147.

Yao JK, Leonard S, Reddy R (2006). Altered glutathione redox state in schizophrenia. Dis Markers 22: 83-93.

Supplementary Information accompanies the paper on the Neuropsychopharmacology website (http://www.nature.com/npp) 